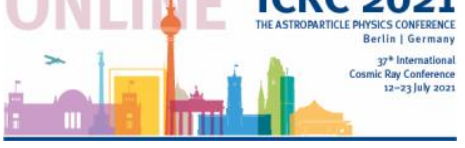

\title{
The Upgrade of Horizon-T Detector
}

\author{
R. U. Beisembaev ${ }^{a}$, E. A. Beisembaeva $a$, O. D. Dalkarov $a$, M. S. Petlenko $b$, V. A. \\ Ryabov $^{b}$, S. B. Shaulov $a$, M. I. Vildanova $a$, V. V. Zhukov $a$ \\ Dmitriy Beznosko $b$,*
}

\section{Shiltsev $c$}

\section{K. A. Baigarin $d$}

\section{T. Kh. Sadykov $e$}

a P. N. Lebedev Physical Institute of the Russian Academy of Sciences, Moscow, Russia

b Clayton State University, Morrow GA 30260, USA

c Fermi National Accelerator Laboratory, IL, USA

d Fermi National Accelerator Laboratory, IL, USA

e Satbayev University, Institute of Physics and Technology, Almaty, Kazakhstan.

E-mail: dmitriybeznosko@clayton.edu

The Horizon-T experiment is located at the elevation of $3346 \mathrm{~m}$ above sea level near the city of Almaty, Republic of Kazakhstan. A thorough comparison of the spatial and temporal characteristics of charged components of Extended Air Showers (EAS) with delayed particles has been conducted between the simulated EAS using CORSIKA simulation package and the selection from the experimental data set of events with two pulses recorded by a detector at $\sim 600 \mathrm{~m}$ distance from axis [1]. This comparison has shown that events with delayed particles cannot be described within existing simulation models. The significance of these results prompted the upgrade of the Horizon-T experiment. New detector points have been added at the $\sim 600 \mathrm{~m}$ to enhance data at that distance. Fast glass-based detector has been added to the scintillator-based detector at center point for accurate measurements of the pulse widths. 


\section{Background of Unusual Cosmic Ray Events}

Cosmic rays have been studied for almost a century. Starting from the mid- $20^{\text {th }}$ century, researchers have observed the pulses in the detectors from Extensive Air Showers (EAS) with the several maxima (or modes) commonly called the showers with delayed particles. Jelley and Whitehouse were the first ones to detects and describe events of this type in 1953 [1]. Until present times, numerous experiments such as [2-6] and others were detecting and studying the EAS events exhibiting this unusual time structures. Every experiment that has undertaken the study of these unusual events had concluded in one form or another that EAS with delayed particles cannot be readily explained using known physical processes. Research of EAS with delayed particles (also called unusual or multimodal events) can help to understand the nature of the cosmic rays with energy of the primary particle above $\sim 10^{16} \mathrm{eV}$ and their interaction with the atmosphere.

\section{Horizon-T Detector Upgrade Motivation}

\subsection{Horizon-T Detector Before Upgrade}

Horizon-T (HT) detector system [7], also sometimes called Horizon-10T or H10T, is a detector system with 10 particle detectors with time resolution on the order of $2 \mathrm{~ns}$ and separated by distances over $1 \mathrm{~km}$ between farthest points. The numeral 10 in the name is used sometimes to explicitly differentiate HT from a series of prototypes that had smaller number of detectors. HT is located at the elevation of $\sim 3400 \mathrm{~m}$ above sea level near the city of Almaty in Kazakhstan. The aerial view and the schematic of the HT detector system are shown in Figure 1. Other technical details can be found in [6] and [7].

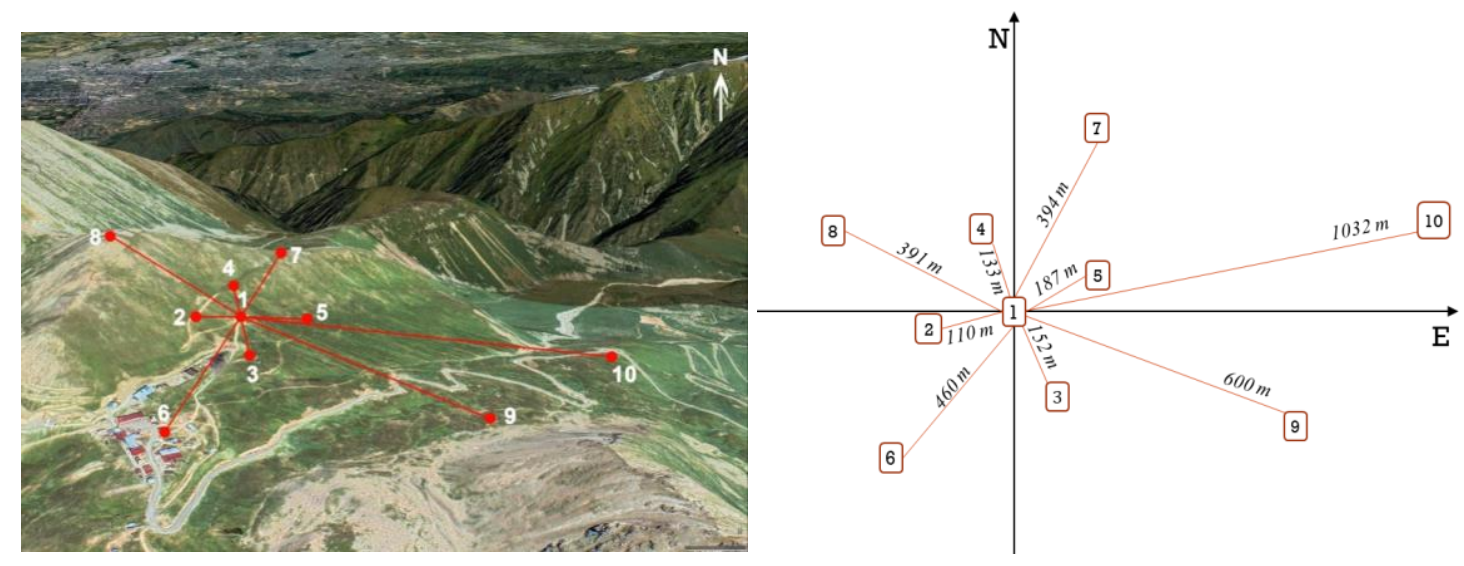

Figure 1: Aerial view of HT detector system (left) and its schematic (right).

\subsection{HT Results}

The unusual events are characterized by the presence of more than one pulse from the charged particles in at least two detectors or more in the event as recorded by HT Data Acquisition (DAQ) system. The example of such even recorded on 2h39m30s March 7, 2018, is presented in Figure 2. This event features the clearly visible multiple pulses from 7 detection points that are correlated between individual detectors. The axis position of this EAS was near detection point 7 and is not shown due to its high amplitude. This event shown an important feature that multiple peaks are clearly separated in the detectors that are $>400 \mathrm{~m}$ away from the core position. 


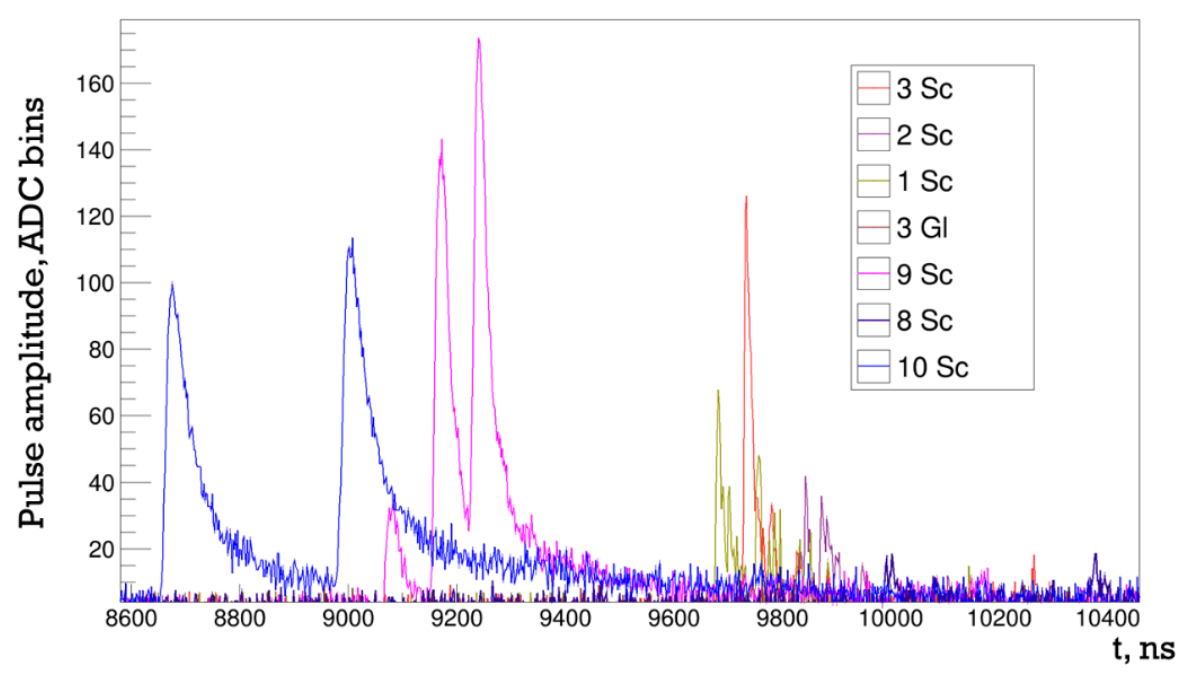

Figure 2: Example of the unusual event

From the existing data sample, over 200 evens were selected that had bimodal signal (e.g. only two pulses) is the detectors that were clearly separated. A plot of the width of these pulses (blue dots - first pulse, red - second pulse) with the simulated EAS disk widths for different energies of the primary particle is shown in Figure 3 [6]. The bimodal events with the clearly separated peaks are best for the analysis and they group at the distances $>400-450 \mathrm{~m}$ from the EAS axis. Therefore, to enhance detection and increase the statistics of bimodal events it was decided to move some the detection points to $\sim 600 \mathrm{~m}$ from center (detection point \#1).

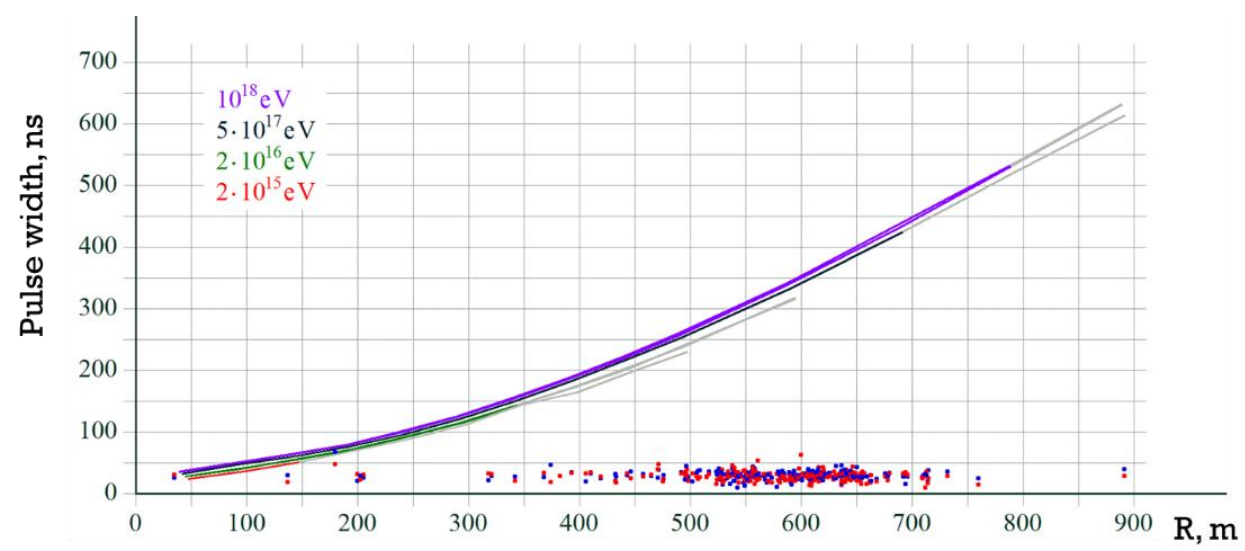

Figure 3: Solid lines - widths of simulated EAS disks for different primary particle energies. Blue dots widths of first peaks in bimodal events, red-second.

\section{Horizon-T Detector After Upgrade}

Following the conclusion from the data from the bimodal event obtained using the HT detector system, detection points 6,7 and 8 were moved to the distance of about $\sim 600 \mathrm{~m}$ from the center of the system, e.g. the detection point 1 . The aerial view and the schematic of this upgraded 
version of the HT detector is presented in Figure 4. This configuration is in effect from Oct 26, 2019, to March 4, 2020.
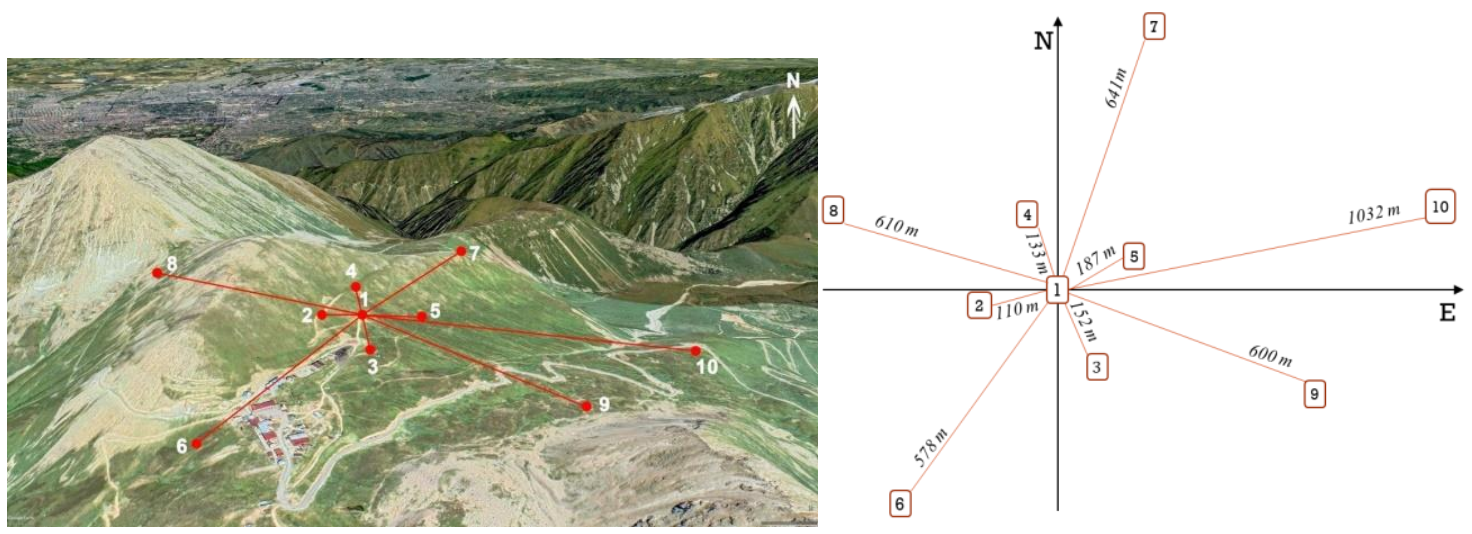

Figure 4: The aerial view (left) and schematic (right) of the HT detector after upgrade.

The coordinates and total distance of each detection point from the HT center (e.g. detection point 1) as well as the PMT (photo multiplier tube) type at each point is shown in Table 1. Note that in points 6-8, the R7723 PMTs were replaced with H6527 on Dec 17, 2019.

Table 1: Coordinates and distance $R$ of each detection point from center (point 1), detector medium and PMT type at each point.

\begin{tabular}{|c|c|c|c|c|c|}
\hline № & $\begin{array}{l}\text { Detector type, } \\
\text { PMT model }\end{array}$ & $\begin{array}{l}\text { X(m) } \\
\text { (North) }\end{array}$ & $\begin{array}{l}Y(\mathbf{m}) \\
\text { (West) }\end{array}$ & $\mathbf{Z}(\mathbf{m})$ & $\mathbf{R}(\mathbf{m})$ \\
\hline 1 & $\mathrm{Sc} 1 \mathrm{~m}^{2}, \mathrm{R} 7723$ & 0.00 & 0.00 & 0.00 & 0.00 \\
\hline 1 & $\begin{array}{l}\text { Glass } 0.62 \mathrm{~m}^{2} \text {, } \\
\text { R7723 }\end{array}$ & 0.00 & 0.00 & 0.00 & 0.00 \\
\hline 2 & $\mathrm{Sc} 1 \mathrm{~m}^{2}, \mathrm{R} 7723$ & -51.84 & 92.80 & 27.60 & 109.82 \\
\hline 3 & $\mathrm{Sc} 1 \mathrm{~m}^{2}, \mathrm{R} 7723$ & -146.58 & -31.46 & -21.50 & 151.45 \\
\hline 4 & $\mathrm{Sc} 1 \mathrm{~m}^{2}, \mathrm{R} 7723$ & 127.76 & 22.86 & 28.50 & 132.88 \\
\hline 5 & Sc $1 \mathrm{~m}^{2}, \mathrm{R} 7723$ & 88.88 & -158.43 & -42.40 & 186.54 \\
\hline 6 & $\mathrm{Sc} 1 \mathrm{~m}^{2}, \mathrm{H} 6527$ & -504.53 & 281.10 & -25.40 & 578.11 \\
\hline 7 & Sc $1 \mathrm{~m}^{2}, \mathrm{H} 6527$ & 578.33 & -272.95 & -42.50 & 640.92 \\
\hline 8 & Sc $1 \mathrm{~m}^{2}, \mathrm{H} 6527$ & 195.03 & 576.91 & 27.60 & 609.61 \\
\hline 9 & $\mathrm{Sc} 1 \mathrm{~m}^{2}, \mathrm{H} 6527$ & -271.87 & -525.76 & -98.60 & 600.05 \\
\hline 10 & $\mathrm{Sc} 1 \mathrm{~m}^{2}, \mathrm{H} 6527$ & 228.36 & -981.14 & -225.30 & 1032.25 \\
\hline
\end{tabular}

For the upgraded configuration, the detector system acceptance and event rate were estimated as well. These are shown in Table 2.

Table 2: Detector system acceptance and event rate estimates.

\begin{tabular}{|c|c|c|c|c|c|c|}
\hline $\mathbf{E}_{\mathbf{0}}[\mathbf{e V}]$ & $\mathbf{1 0}^{\mathbf{1 6}}$ & $\mathbf{2} \cdot \mathbf{1 0}^{\mathbf{1 6}}$ & $\mathbf{5} \cdot \mathbf{1 0}^{\mathbf{1 6}}$ & $\mathbf{1 0}^{\mathbf{1 7}}$ & $\mathbf{2} \cdot \mathbf{1 0}^{\mathbf{1 7}}$ & $\mathbf{1 0}^{\mathbf{1 8}}$ \\
\hline $\boldsymbol{\Gamma}\left[\mathbf{k m}^{\mathbf{2}} \mathbf{s r}\right]$ & 0.38 & 0.72 & 0.97 & 1.54 & 2.74 & 6.37 \\
\hline $\mathbf{N} / \mathbf{t}[\mathbf{e v e n t} / \mathbf{h}]$ & 25.60 & 7.98 & 2.25 & 1.05 & 0.53 & 0.07 \\
\hline
\end{tabular}




\section{Conclusion}

It is evident from the simulations and current physics understanding that EAS should be a single disk with statistical variations within it. The disk thickness increases from axis outwards; particle density in the disk decreases from axis outwards and any large detectable variations should be extremely rare. This should produce the response in the particle detectors as a single pulse of width and area corresponding to the disk thickness and density. However, HT detector system has detected numerous events that are of unusual structure (typically called 'events with the delayed particle'). After initial data analysis, HT detector has been optimized to maximize detection of such events with high peak separation.

For the future plans, using the upgraded Horizon-T detector system, we plan to continue the detailed study of the unusual EAS events.

\section{References}

[1] J V Jelley and W J Whitehouse. 1953 Proc. Phys. Soc. A 66 (454), 1953

[2] J. Linsley and L. Scarsi. Phys. Rev. 128 (2384), 1962

[3] Baxter A.J., Watson A.A., Wilson J.G. Proc. 9 ICRC. 2 (724), 1965

[4] H. Sakuyama, N. Suzuki, and K. Watanabe. Nuovo Cim. A 78 (147), 1983

[5] Fomin Yu.A., Garipov G.K. et al., Proc. 28 ICRC. 1 (973), 2003

[6] R. Beisembaev et al., Spatial and Temporal Characteristics of EAS with Delayed Particles, in proceedings of POS ( ICRC2019) 195

[7] R. Beisembaev et al., Extensive Air Showers with Unusual Spatial and Temporal Structure, in proceedings of EPJ Web of Conferences 208, 06002 (2019) 